This item was submitted to Loughborough's Research Repository by the author.

Items in Figshare are protected by copyright, with all rights reserved, unless otherwise indicated.

\title{
On the theory of standing waves in tyres at high vehicle speeds
}

PLEASE CITE THE PUBLISHED VERSION

http://dx.doi.org/10.1016/j.jsv.2010.05.001

PUBLISHER

(c) Elsevier

VERSION

AM (Accepted Manuscript)

LICENCE

CC BY-NC-ND 4.0

REPOSITORY RECORD

Krylov, Victor V., and O. Gilbert. 2012. "On the Theory of Standing Waves in Tyres at High Vehicle Speeds". figshare. https://hdl.handle.net/2134/10048. 
This item was submitted to Loughborough's Institutional Repository (https://dspace.lboro.ac.uk/) by the author and is made available under the following Creative Commons Licence conditions.

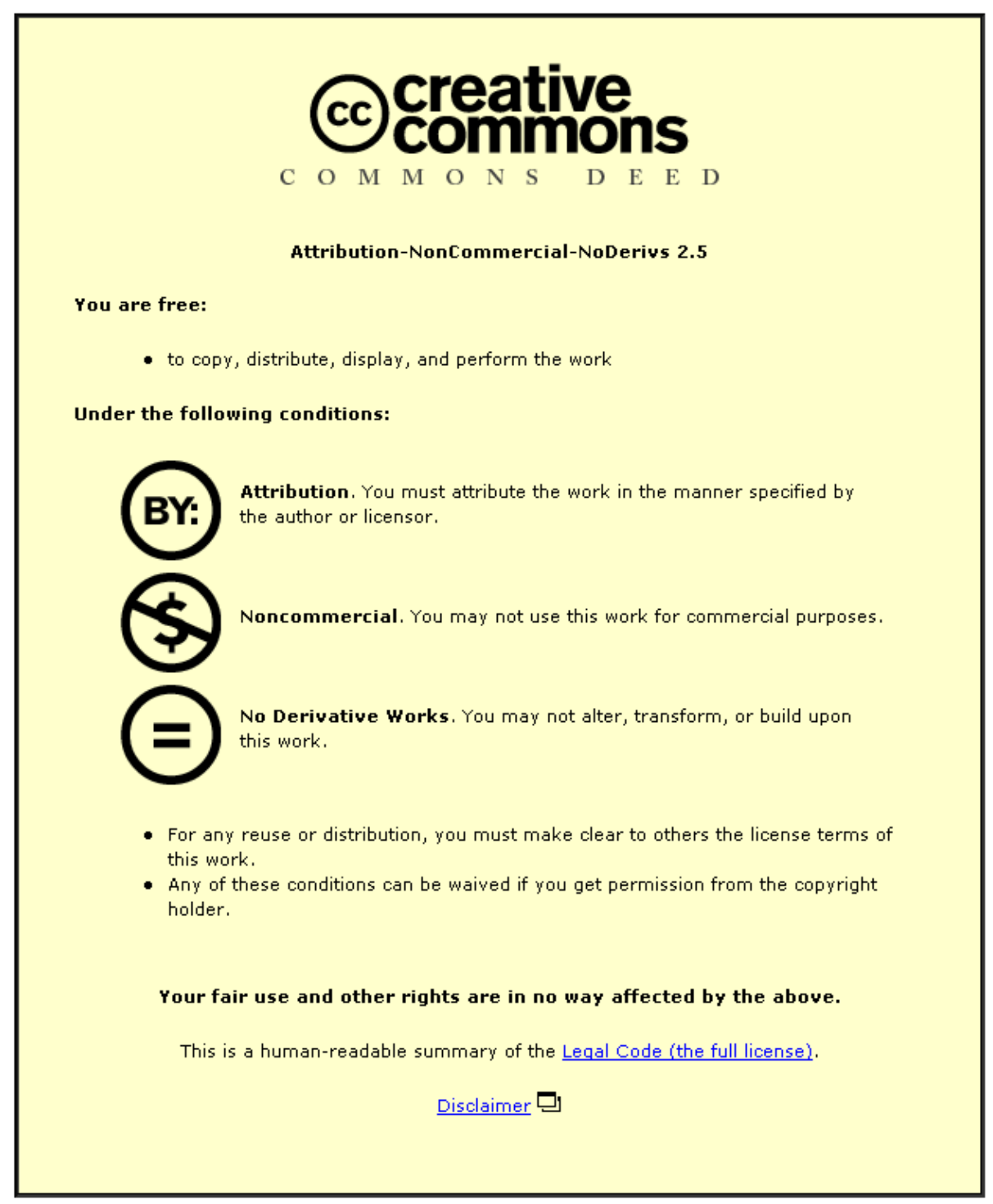

For the full text of this licence, please go to: http://creativecommons.org/licenses/by-nc-nd/2.5/ 


\title{
On the theory of standing waves in tyres at high
}

\section{vehicle speeds}

\author{
Victor V. Krylov*, Oliver Gilbert \\ Department of Aeronautical and Automotive Engineering, \\ Loughborough University, \\ Loughborough, Leicestershire LE11 3TU, UK
}

\section{Abstract}

When a vehicle moves at high speed and if the speed exceeds a certain 'critical velocity', then quite intensive flexural waves travelling around the circumference of the tyre emerge from the trailing edge of the contact patch. To the observer these waves in the tyre appear to be stationary. Therefore, in the literature they are often referred to as 'standing waves in tyres'. Although this phenomenon has been studied since the 1950s and a number of successful predictions of experimentally observed features had been

\footnotetext{
* Corresponding author. Tel.: +44 1509 227216;

E-mail address: V.V.Krylov@lboro.ac.uk
} 
made, it is still not well understood. In the present paper, a new physically explicit theory of tyre standing waves has been developed. The theory is based on a tensioned beam model of a tyre. Simple analytical expressions have been derived for the amplitudes and shapes of generated waves and for the most important parameter characterising the phenomenon of tyre standing waves - the vehicle critical speed beyond which the standing waves occur. The performance of the theory is examined by varying the tyre parameters and by comparing the results, where possible, to other similar work. The obtained predictions show that the developed theory explains and predicts the phenomenon of tyre standing waves surprisingly well, given its simplicity and the uncertainty in the tyre parameters used in calculations. It also has the potential for further development.

Keywords: Tyre standing waves, Critical vehicle speed, Tyre vibration theory, Tensioned beam model.

\section{Introduction}

The phenomenon of standing waves in tyres occurs at high speeds, when a vehicle speed exceeds a certain 'critical velocity'. Then quite intensive flexural waves travelling around the circumference of the tyre emerge from the trailing edge of the contact patch. To the stationary observer these waves in the tyre appear to be stationary, but they are in fact travelling around the tyre at the same speed as the rotation of the tyre. This is why 
these waves are often referred to in the literature, not quite correctly, as 'standing waves in tyres'. Since this term is already well established, we will use it as well.

The large displacements of the tyre surface due to the generated standing waves introduce a large amount of energy into the tyre material, which can lead to a quick tyre overheating and failure, or at the very least can severely affect the handling of the vehicle. Since the phenomenon of standing waves is usually observed at high vehicle speeds, clearly aircraft tyres, race car tyres and to a lesser extent passenger car tyres are likely to experience it. Note that manufactured tyres normally have a speed rating to indicate the experimentally established maximum vehicle speeds below which they can be used safely. It is indicated by the letter-symbol on the tyre sidewall at the end of a series of numbers. For example, the T rating identifies speeds up to $190 \mathrm{~km} / \mathrm{h}$, and the $\mathrm{V}$ rating means speeds up to $240 \mathrm{~km} / \mathrm{h}$. However, the real vehicle speeds at which the standing waves occur can be substantially lower due to poor maintenance of the tyre and/or due to its under-inflation as well as because of other conditions, such as the load on the tyre.

The phenomenon of tyre standing waves has been studied since the 1950s [1,2], and a useful review of early experimental and theoretical works (published before 1970) can be found in the paper of Ames [3]. Starting from the work of Turner [2], there have been many attempts at theoretical modelling of this phenomenon (see also [3] and references therein). However, for the sake of simplicity, in most of the early works the authors did not consider the phenomenon of standing waves in tyres as wave generation problem, but rather argued from a wave propagation viewpoint. The common approach was to employ differential equations for free wave propagation in different simplified models, usually membrane models, and to use the fact that in the case of emergence of a stationary wave all time derivatives in the equations have to be zero. As a result, one 
can obtain an algebraic equation whose roots give an expression for a critical vehicle speed at which standing waves occur. Although this approach can be useful in predicting critical vehicle speeds, it is quite unsatisfactory and incomplete as it does not describe generated waveforms or the effect of tyre load.

More recently, Chatterjee et al [4] undertook model experiments using a counterrotating drum to investigate the behaviour of a small-scale balloon tyre inflated to several different pressures. Although the balloon tyres used are not much relevant to real automotive tyres, they were convenient to measure generated waveforms at several different angular velocities and to observe the formation of standing waves. Saran et al [5] reported the results of laboratory measurements for determining the rolling dynamic stiffness and damping coefficients of small-sized bias-ply tyres.

With the advancement of computer technology and finite element techniques it has become possible to create sufficiently accurate numerical models capable to describe the phenomenon of tyre standing waves rather well. However, there is a question as to how useful the numerical results would be without understanding the physical mechanisms responsible for generation of tyre standing waves and their behaviour at different values of tyre parameters. To achieve such understanding the authors of papers describing different numerical approaches are forced to resort to analytical or semianalytical theories anyway, and it is important what theories they choose to find an adequate interpretation of their results.

Note that the authors of the paper [4] also give a theoretical interpretation of their experimental results. However, the theoretical part in [4] seems to be rather controversial. In particular, the authors describe a balloon tyre by a one-dimensional beam-string model that does not fit naturally to an essentially two-dimensional geometry of this configuration (there are no tyre belts in balloon tyres). Also, they 
consider the contact boundary problem in their model. As a result, the model becomes overly complicated, with mixed boundary conditions, and requires numerical solution. Apparently, this complexity influenced the authors' interpretation of the phenomenon of tyre standing waves, which they associated specifically with the nonlinear contact problem. In particular, the authors state that the properties of tyre standing waves "are determined solely by the lift-off conditions at the trailing contact edge” [4].

Another well-known theoretical approach to the phenomenon of tyre standing waves is the so-called resonance approach [6,7]. This approach is based on the inherent circular periodicity of a tyre, in particular if a tyre belt is modelled as a thin ring and the side walls are modelled as an elastic foundation. Taking this periodicity into account, Padovan [6] has developed a theory that has given a resonance interpretation of the phenomenon of tyre standing waves. He has included viscoelasticity into his ring model as well as the Coriolis effect. One of the results of his work shows that at the critical speed without damping the tyre response is completely symmetrical about the contact patch. Increasing the level of damping, however, moves the wave or retards the response to behind the contact patch. Another positive result is the fact that the waves attenuate quite significantly before reaching the leading edge of the contact patch, just as they do in real tyres. Soedel [7] applied the modal expansion approach to a ring model and also used a resonance approach. To enable a more accurate prediction of the critical speed he required modal damping factors that could be found by experimentation.

Note that the above-mentioned resonance approach is good enough at predicting the standing wave generation. However, several aspects of this approach do not conform naturally to the experimental observations. Indeed, if not to take into account vibration energy losses in the tyre, then the increase in vehicle speed above critical, according to 
the modal resonance approach, would result in discrete (resonance) changes in the waveforms of standing waves, which is not confirmed by experimental observations. However, the inclusion of energy losses improves the situation (see [6]), describing a rather smooth evolution of standing wave forms at vehicle speeds that are above the critical speed.

Contrary to the authors of [6,7], we believe that the phenomenon of tyre standing waves is not a resonance phenomenon by nature, although its description in terms of tyre resonant vibration modes is, of course, valid (as in [6,7]). However, this resonant description is somewhat similar to a modal approach to sound propagation between two locations in a large room, where acoustic resonances associated with neighbouring modes of the room overlap with each other due to the presence of acoustic dissipation and thus do not reveal themselves. It is well known therefore that using a much simpler free field approach to sound propagation between the above-mentioned two locations in a large room gives the same result as a much more complex modal approach. In our opinion, using the resonance approach [6,7] to describe tyre standing waves brings unnecessary complications to the theory and masks the real physics of the phenomenon.

In addition to the above-mentioned papers directly concerned with standing waves in tyres, there are many investigations dealing with different aspects of tyre dynamics with applications to tyre-generated noise. In particular, Kropp [8] used a model of a tyre as a thin ring on an elastic foundation to investigate a tyre response to a harmonic force excitation. He showed that the behaviour of his ring model was string-like below $250 \mathrm{~Hz}$ and beam-like between $250 \mathrm{~Hz}$ and $400 \mathrm{~Hz}$. Pinnington et al [9] derived a wave equation for an infinite flattened tyre belt, and considered shear and tension as well as bending. The aim of this work was to develop a model to describe the vibration behaviour of tyres over a range of frequencies and investigate their noise generation characteristics. 
This necessitated the examination of the tyre behaviour along the cross section of the tyre. A smooth uniform belt was assumed and the modes of vibration across the cross section were identified. Muggleton et al [10] developed a tyre model comprising a central tensioned plate supported by two equal side plates. Again, the aim of this paper was to describe the behaviour of tyres over a range of frequencies for the purpose of noise prediction. The experiments conducted on a real inflated tyre have shown reasonable agreement with the theoretical predictions. The aim of Perisse' work [11] was to investigate the distribution of tyre vibration energy in front and in the rear of the contact patch, again for the purpose of tyre/road noise prediction.

It can be seen from the above that there are many different approaches to the modelling of tyre dynamic properties. This is particularly true for the phenomenon of tyre standing waves that is still not well understood in spite of a number of successful predictions of experimentally observed features. So far, no one theory gives the preferred approach, which demonstrates that research into this problem is far from being finished.

The aim of the present paper is to develop a new physically explicit analytical theory of tyre standing waves based on a one-dimensional model of infinite tensioned beam on an elastic foundation subjected to a constant moving load. The main objective of such theory is to identify the most important physical mechanisms of tyre standing wave generation and to analyse the vehicle critical speed as a function of different physical and geometrical parameters of the tyre. The reasons for using this approach are that the above-mentioned infinite tensioned beam model, being physically explicit, represents the phenomenon of tyre standing waves surprisingly well and has the potential for further development. The main conceptual idea of this theory is that generation of tyre standing waves is of the same physical nature as generation of Mach 
waves in acoustics, Cherenkov waves in optics, water waves from moving ships in hydrodynamics, and track and ground waves from high speed trains in railway engineering, not mentioning others. Simple analytical expressions are derived for the amplitudes and shapes of generated tyre standing waves and for the most important parameter characterising the phenomenon - the vehicle critical speed beyond which the standing waves occur.

\section{Outline of the theory}

To describe generation of tyre standing waves we employ a simple model comprising a concentrated force of constant magnitude moving at constant speed along a finite length of an infinite tensioned Euler - Bernoulli beam resting on an elastic Winkler foundation (see Fig. 1). Obviously, the beam represents a tyre belt, and the role of the elastic foundation is to describe the effect of tyre sidewalls. The inclusion of tension into the beam model is needed to describe the tyre tension resulting from the air inflation pressure.

Note that a beam without tension resting on an elastic foundation has been used widely in railway engineering for modelling a railway track supported by the ground. It is well known that such a model can describe generation of high-amplitude track bending waves (also known as bow waves) by train wheels moving at constant speed $c$ if this speed is larger than the so-called minimal phase velocity of track bending waves [12]. Under this condition, the generated track bending waves propagate with the same speed $c$ and therefore appear to be stationary in respect of the contact patch of a moving wheel. 
The main idea to be developed and examined in the present paper is that the phenomenon of tyre standing waves is physically very similar to the above-mentioned phenomenon of bow waves in railway tracks. However, instead of a constant load force applied to a railway track from a moving train wheel, the force applied to a rotating tyre belt is represented by a constant ground reaction force transmitted through the contact patch that moves along a tyre circumference with the speed $c$ of a vehicle. The curvature of a tyre belt is assumed to be nonessential from the point of view of tyre standing wave generation and propagation. This assumption is based on the well-known fact following from the symmetry consideration, namely that changes in velocities of out-of-plane waves in curved beams are rather small as they are proportional to $R^{-2}$, where $R$ is the radius of curvature. However, the curvature is important for calculation of the tyre belt tension at a given air pressure.

Before considering the above-mentioned model of an infinite tensioned beam on an elastic foundation to describe generation of tyre standing waves, let us first recall some of the well known facts from the theory of an infinite elastically supported beam without tension that is used in railway engineering.

The differential equation describing flexural vibrations of an infinite untensioned beam on an elastic foundation subjected to a constant force $P$ moving at constant speed $c$ has the following form (see e.g. [12] )

$$
E J \frac{\partial^{4} v(x, t)}{\partial x^{4}}+\mu \frac{\partial^{2} v(x, t)}{\partial t^{2}}+2 \mu \omega_{b} \frac{\partial v(x, t)}{\partial t}+k v(x, t)=P \delta(x-c t)
$$

Here $v(x, t)$ is the beam deflection at point $x$ and time $t, E$ is the Young's modulus of the beam material, $J$ is the second moment of area of the beam, $\mu$ is the beam 
mass per unit length, $k$ is stiffness of the elastic foundation, $\omega_{b}$ is the parameter describing damping in the beam. The right-hand side of the above equation includes the force magnitude $P$ and Dirac's delta-function $\delta(x-c t)$ describing a unit concentrated force.

It is convenient to introduce the non-dimensional variable, $s=\lambda(x-c t)$, which reflects the fact that its origin moves together with the load at uniform speed $c$. Here $\lambda=\left(\frac{k}{4 E J}\right)^{\frac{1}{4}}$. Replacing differentiation over $x$ and $t$ in Eqn (1) by differentiation over the non-dimensional variable $s$ and taking into account that

$$
\frac{\partial s}{\partial x}=\lambda \quad \text { and } \quad \frac{\partial s}{\partial t}=-\lambda c
$$

one can arrive from Eqn (1) to the ordinary differential equation [12]

$$
\frac{d^{4} v(s)}{d s^{4}}+4 \alpha^{2} \frac{d^{2} v(s)}{d s^{2}}-8 \alpha \beta \frac{d v(s)}{d s}+4 v(s)=8 \bar{\delta}(s)
$$

where

$$
\begin{gathered}
v(s)=v(x, t) \frac{1}{v_{0}}, \quad v_{0}=\frac{P \lambda}{2 k}, \\
\alpha=\frac{c}{c_{c r}}=\frac{c}{2 \lambda}\left(\frac{\mu}{E J}\right)^{\frac{1}{2}}, \quad c_{c r}=2 \lambda\left(\frac{E J}{\mu}\right)^{\frac{1}{2}}, \quad \beta=\left(\frac{\mu}{k}\right)^{\frac{1}{2}} \omega_{b} \text { and } \bar{\delta}(s)=\frac{1}{\lambda} \delta(x) .
\end{gathered}
$$


The formal solution of Eqn (3) in terms of the non-dimensional variable $s$ can be represented in the following integral form [12]:

$$
v(s)=\frac{4}{\pi} \int_{-\infty}^{\infty} \frac{e^{i s q}}{q^{4}-4 \alpha^{2} q^{2}-i 8 \alpha \beta q+4} d q,
$$

where $q$ is a variable of integration. The result of the integration can be obtained in the complex $q$ - plane, by the method of residues, where the poles of the integrand in Eqn (6) are determined by the roots of the denominator $Q(q)$ :

$$
Q(q)=q^{4}-4 \alpha^{2} q^{2}-i 8 \alpha \beta q+4=0
$$

The analysis of Eqns (4) - (6) shows that different approximate solutions for $v(s)$ can be obtained for different values of the parameters $\alpha$ and $\beta$. In particular, the following four damping cases can be considered: No damping: $\beta=0$, Light damping: $\beta<<1$, Critical damping $\beta=\beta_{c r}$, and Super-critical damping $\beta>\beta_{c r}$.

Here we will be only concerned with the most important case of light damping: $\beta<<1$. For this case, one can consider the following speed conditions:

(i) Sub-critical: $\alpha<1$,

(ii) Critical: $\alpha=1$,

(iii) Super-critical: $\alpha>1$. 
The latter condition, that corresponds to a load motion with a speed $c$ larger than the so-called minimal phase velocity or critical wave speed of flexural waves for the beam, $c_{c r}=2 \lambda\left(\frac{E J}{\mu}\right)^{1 / 2}$, is associated with the generation of waves propagating along the beam with the speed of the load motion, $c$, or bow waves.

Physically, this wave generation mechanism is the same as the one responsible for a sonic boom from supersonic aircraft (Mach waves) [13] or for a ground vibration boom from high-speed trains [14], as well as for Cherenkov waves in optics, and for water waves from moving ships in hydrodynamics. Applied to tyres, this mechanism would imply that tyre waves are generated when the vehicle speed is larger than a certain critical speed of the tyre flexural waves. Since these waves propagate along the tyre circumference with the velocity of the moving force (acting from the ground at the contact patch), they appear for a stationary observer as stationary, or 'standing' waves. This, of course, is not a very precise term as the waves are in fact propagating along the tyre perimeter.

The solution for $v(s)$ has different forms for $\mathrm{s}<0$ and $\mathrm{s}>0$ [12]:

$$
\begin{aligned}
& v(s)=\frac{2}{a_{2}\left(D_{3}^{2}+D_{4}^{2}\right)} e^{b s}\left(D_{3} \cos a_{2} s-D_{4} \sin a_{2} s\right), \quad \text { for } s<0, \\
& v(s)=\frac{2}{a_{1}\left(D_{1}^{2}+D_{2}^{2}\right)} e^{-b s}\left(D_{1} \cos a_{1} s+D_{2} \sin a_{1} s\right), \quad \text { for } s>0 .
\end{aligned}
$$

Here 


$$
D_{1}=a_{1} b, \quad D_{2}=b^{2}-\frac{1}{4}\left(a_{1}^{2}-a_{2}^{2}\right), \quad D_{3}=a_{2} b, \text { and } \quad D_{4}=b^{2}+\frac{1}{4}\left(a_{1}^{2}-a_{2}^{2}\right) \text {, }
$$

where for a super-critical speed condition that we are interested in $(\alpha>1, \beta<<1)$

$$
b \approx \frac{\alpha \beta}{\left(\alpha^{4}-1\right)^{\frac{1}{2}}}, \quad \text { and } \quad a_{1,2} \approx\left(\alpha^{2}+1\right)^{\frac{1}{2}} \pm\left(\alpha^{2}-1\right)^{\frac{1}{2}}
$$

To model tyre standing waves, which is the aim of the present paper, let us now include the effect of tyre tension, in addition to the above-mentioned effect of bending stiffness EJ. To do this, we recall the well-known membrane (string) equation

$$
\mu \frac{\partial^{2} v}{\partial t^{2}}-S \frac{\partial^{2} v}{\partial x^{2}}=0
$$

where $S$ is the tension force. Comparing Eqn (12) with Eqn (1), one can see that the additional term $-S \frac{\partial^{2} v}{\partial x^{2}}$ should be added to the left-hand side of Eqn (1) to include the tension. This results in the following combined differential equation that will be used in further analysis:

$$
E J \frac{\partial^{4} v(x, t)}{\partial x^{4}}+\mu \frac{\partial^{2} v(x, t)}{\partial t^{2}}-S \frac{\partial^{2} v(x, t)}{\partial x^{2}}+2 \mu \omega_{b} \frac{\partial v(x, t)}{\partial t}+k v(x, t)=P \partial(x-c t)
$$


To solve Eqn (13) one can use the same approach that has been described above for the solution of Eqn (1). Indeed, by replacing differentiation over $x$ and $t$ in Eqn (13) by differentiation over the non-dimensional variable $s=\lambda(x-c t)$ and by taking into account Eqn (2), one can arrive at the following ordinary differential equation:

$$
\frac{d^{4} v(s)}{d s^{4}}+4 \tilde{\alpha}^{2} \frac{d^{2} v(s)}{d s^{2}}-8 \tilde{\alpha} \tilde{\beta} \frac{d v(s)}{d s}+4 v(s)=8 \bar{\delta}(s) .
$$

Obviously, Eqn (14) has the same form as Eqn (3). The only difference is that instead of the parameters $\alpha$ and $\beta$ that are present in Eqn (3) (as well as in the resulting Eqns (4)- (11)) we have introduced the two modified parameters, $\tilde{\alpha}, \widetilde{\beta}$, taking into account the tyre tension:

$$
\begin{gathered}
\tilde{\alpha}=\alpha \sqrt{1-\frac{S}{\mu c^{2}}} \\
\tilde{\beta}=\frac{\beta}{\sqrt{1-\frac{S}{\mu c^{2}}}}
\end{gathered}
$$

Using the corresponding modified critical speed condition $\tilde{\alpha}=1$, it is easy to show that the new critical speed $c_{c r i t}$ of a beam (tyre) taking the tension into account is

$$
C_{c r i t}=\sqrt{{C_{c r}{ }^{2}+C_{m e m}{ }^{2}}^{2}} \text {, }
$$


where $\quad c_{c r}=2 \lambda\left(\frac{E J}{\mu}\right)^{1 / 2}$ is a critical wave speed in a beam without tension and $C_{\text {mem }}=\left(\frac{S}{\mu}\right)^{1 / 2}$ is the velocity of membrane (string) waves.

The wave form solution for the super-critical speed condition, $\tilde{\alpha}>1, \tilde{\beta}<<1$, is described by the same equations (8) - (10). However, the parameters $b$ and $a_{1,2}$ are now described by the following expressions:

$$
\begin{gathered}
b \approx \frac{\tilde{\alpha} \tilde{\beta}}{\left(\tilde{\alpha}^{4}-1\right)^{\frac{1}{2}}}=\frac{\alpha \beta}{\left(\alpha^{4}\left(1-\frac{S}{\mu c^{2}}\right)^{2}-1\right)^{\frac{1}{2}}}, \\
a_{1,2} \approx\left(\tilde{\alpha}^{2}+1\right)^{\frac{1}{2}} \pm\left(\tilde{\alpha}^{2}-1\right)^{\frac{1}{2}}=\left(\alpha^{2}\left(1-\frac{S}{\mu c^{2}}\right)+1\right)^{\frac{1}{2}} \pm\left(\alpha^{2}\left(1-\frac{S}{\mu c^{2}}\right)-1\right)^{\frac{1}{2}} .
\end{gathered}
$$

Note that, using Eqn (17), the parameter $\tilde{\alpha}$ can be also expressed as

$$
\tilde{\alpha}=\frac{c}{C_{c r i t}}=\frac{c}{\sqrt{c_{c r}^{2}+C_{\text {mem }}^{2}}}
$$

\section{Results of the calculations and discussion}




\subsection{Choice of tyre parameters}

In the present paper, most of the required tyre parameters used for example calculations have been adopted from the paper of Muggleton et al [10] where they have been determined experimentally. One parameter, stiffness of the equivalent elastic foundation that was not measured in [10], has been taken from the paper of Kropp [8]. All the parameters used for calculations are shown in Table 1.

Tyre tension $S$ applied to the above-mentioned tensioned beam model has been calculated according to the well-known formula $S=p R b[\mathrm{~N}]$ following from the statics equilibrium condition. Here $p$ is the tyre air pressure, $R$ is the tyre radius and $b$ is the tyre belt width. The value of air pressure $p=206850 \mathrm{~Pa}$ (or $30 \mathrm{psi}$ ) typical for passenger car tyres has been used in most of the calculations. Mass of the tyre belt per unit length $\mu$ has been calculated as $\mu=\rho h b[\mathrm{~kg} / \mathrm{m}]$, where $\rho$ and $h$ are the tyre belt mass density and thickness respectively. The second moment of area $J$ has been

calculated as $J=h^{3} b / 12\left[\mathrm{~m}^{4}\right]$, and for the non-dimensional beam damping factor $\beta$ a typical value of 0.2 has been chosen.

\subsection{Calculated critical velocities and waveforms}

For the tyre parameters listed in Table 1, one can calculate:

$$
\begin{aligned}
& \lambda=\left(\frac{k}{4 E J}\right)^{\frac{1}{4}}=18.55 \mathrm{~m}^{-1}, \quad C_{c r}=2 \lambda\left(\frac{E J}{\mu}\right)^{1 / 2}=27.09 \mathrm{~m} / \mathrm{s}, \text { and } \\
& C_{\text {mem }}=\left(\frac{S}{\mu}\right)^{1 / 2}=56.85 \mathrm{~m} / \mathrm{s} .
\end{aligned}
$$


Then, using equation (17), one obtains the following value of the tyre critical speed:

$$
C_{\text {crit }}=\sqrt{{C_{c r}{ }^{2}+{C_{\text {mem }}}^{2}}^{2}}=62.98 \mathrm{~m} / \mathrm{s} \text { or } 226.72 \mathrm{~km} / \mathrm{h} \text {. }
$$

This result, being in the range of typical values of critical speeds for high quality passenger car tyres, seems to be satisfactory, considering the uncertainty of the tyre parameters used for calculations and simplicity of the model at this stage of development. In particular, it is of a comparable magnitude to the expected critical speed of a particular radial tyre [10] used for the above calculations, which is speculated to be between $200 \mathrm{~km} / \mathrm{h}$ and $250 \mathrm{~km} / \mathrm{h}$. Unfortunately, no precise critical speed information was available for the particular tyre in question.

Figure 2 illustrates a typical form of tyre standing wave calculated using Eqns (8) (10) and (18), (19) for the parameters mentioned in Table 1 and for the load (vehicle) speed of $98 \mathrm{~m} / \mathrm{s}$ (or $352.8 \mathrm{~km} / \mathrm{h}$ ), which corresponds to the super-critical speed condition: $\tilde{\alpha}=1.556$. It can be seen that the tyre standing wave is generated mainly at the trailing edge of the contact patch (for $s<0$ ), and it attenuates quickly with the distance from the contact patch. To illustrate the generated standing waves more explicitly, using a real dimension of tyre circumference, a calculated polar plot is shown in Fig. 3.

Note that the similarity of the standing wave forms shown in Figs. 2 and 3 to those observed in tyres, in particular to the experimental standing waveforms observed by Chatterjee et al [4], is quite obvious. It is interesting that even the small ripples in front of the contact patch, that are clearly seen in Fig. 2 (albeit not in Fig. 3, because of the small scale for radial displacements in the latter), reproduce the feature experimentally 
observed in [4]. These small ripples have also been predicted by the authors of [4] using their numerical model.

The effect of vehicle speed on generated waveforms at super-critical speed condition is illustrated in Fig. 4 for the values of vehicle speed of $98 \mathrm{~m} / \mathrm{s}(352.8 \mathrm{~km} / \mathrm{h})$, $120 \mathrm{~m} / \mathrm{s}(432 \mathrm{~km} / \mathrm{h})$ and $142 \mathrm{~m} / \mathrm{s}(511.2 \mathrm{~km} / \mathrm{h})$. It can be seen that for larger vehicle speeds the spatial period of oscillations increases. This is in line with numerous experimental observations and with the results of Padovan [6] obtained using the resonance approach.

The effect of damping on calculated forms of tyre standing waves under supercritical speed condition is shown in Fig. 5 for the vehicle speed of $352.8 \mathrm{~km} / \mathrm{h}$ and for the values of normalised damping $\beta$ equal to $0.1,0.2$ and 0.3 . It is seen that the increase in damping results in a more rapid decay of the waveforms with the distance from the contact patch, which again agrees with the results of Padovan [6].

\subsection{Effects of different tyre parameters on critical speed}

To validate the tensioned beam model of tyre standing waves developed in the previous sections it is important to understand its behaviour for variable tyre parameters. This section deals with variations of some of the parameters reflecting actual operating conditions as well as changes with a tyre throughout its life and describes their effects on the critical speed.

\subsubsection{Effect of tyre pressure on critical speed}


Note that increasing the tyre pressure $p$ is essentially the same as increasing the tyre belt tension $S$ due to the above-mentioned linear relationship between the tyre pressure and the tyre tension: $S=p R b$. Figure 6 shows that the critical speed $c_{c r i t}$ increases with pressure. Obviously, the relationship only slightly differs from linear.

It should be mentioned that the tension term is dominant for the tyre parameters given in Table 1. The contribution of the elastic foundation and of the tread belt bending stiffness on the critical speed is relatively small, as can be seen if the two speeds, $c_{m e m}$ and $c_{c r}$, contributing to the critical speed are plotted alongside $c_{c r i t}$ (see Fig. 7).

Note that in the tensioned beam model of a tyre considered in this paper it was assumed that the stiffness of the equivalent elastic foundation, describing the reaction of the tyre sidewalls, does not alter with the air pressure. The air pressure in a tyre, however, does effect the stiffness of the foundation, as well as the tension. This is a drawback of the present model that could be mitigated in a future work.

\subsubsection{Effect of tread thickness on critical speed}

This part of the investigation relates to the wear that occurs under the normal working life of the tyre and results in reduction of the belt thickness. Figure 8 shows how the model predicts the critical speed for varying thickness (depth) of a tread belt.

It is seen from Fig. 8 that as the tread thickness decreases the critical speed of wave propagation increases. The reason for this is that for medium and small values of tread thickness the tension term is dominant and is dependent upon the mass per unit length of the belt $\mu$. Decreasing the thickness of the belt reduces the mass per unit length, thus increasing the membrane wave propagation speed. In effect, the tyre belt tends towards a membrane as the tread thickness decreases. 
Figure 9 demonstrates how both constituents of the critical speed in Eqn (17) are affected by the change in tread thickness. One can see that the membrane term is indeed dominant for small and medium values of thickness, but as the tread depth increases the beam bending stiffness has more of an influence due to the effect of the second moment of area $J$. This is due to the fact that the mass per unit length, $\mu$, has a linear relationship with the depth of the tread, $h$, whilst the second moment of area, $J$, is governed by a third order relationship with the depth, which makes it more influential for larger $h$. Note that for very large values of tyre thickness (above about $0.044 \mathrm{~m}$ ), that are beyond the range typical for car tyres, the beam bending stiffness term becomes dominant and the resulting critical speed becomes almost independent of the belt thickness.

In practical terms, the above results mean that if a driver were to neglect the maintenance of the tyres and the tread were to decrease in depth due to wear, then the critical speed of the tyre would increase, thus reducing the risk of tyre waves to occur. Taking the onset of tyre standing wave generation as the only concern, then ignoring tyre wear is clearly not as important as maintaining air pressure.

\section{Conclusions}

The main conclusion following from this paper is that the infinite tensioned beam theory of tyre standing waves gives a clear physical explanation of the wave generation mechanism and proves to be a good approximation to the real phenomenon, with the potential for improvements in a number of areas. 
The model shows a close physical likeness of the predicted waveforms to the ones observed in experiments, although this should always remain a secondary consideration as the main concern is the critical speed prediction.

Considering the model's simplicity and the fact that some of the tyre parameters used for calculations may be not very precise, the results of the critical speed prediction give the right order of magnitude.

The precision of the results for tyre critical speeds can be further improved by interpreting the values of the model parameters more adequately. In particular, the mass per unit length, $\mu$., is the most likely parameter to be wrong, and it has a large effect on the results.. Another parameter that could be re-examined is stiffness of the equivalent elastic foundation $k$, in particular its dependence on tyre pressure that has been ignored in this study. There is a potential for future work to quantify these parameters more accurately.

Among other effects that could be considered in order to improve the accuracy of the prediction are the effect of rotational velocity on the belt tension and the effect of non-linear elasticity associated with tyre loading. 


\section{References}

[1] E.R. Gardner, T. Worswick, Behaviour of tyres at high speed, Trans. Inst. Rubber Ind., 27 (1951) 127-146.

[2] D.M. Turner, Wave phenomena in tyres at high speed, Proc. Rubber Technol. Conf. London, (1954) 735-748.

[3] W.F. Ames, Waves in tyres: Part I: Literature review on travelling waves, Textile Research Journal, 40 (1970) 498-503.

[4] A. Chatterjee, J.P. Cusumano, J.D. Zolock, On contact-induced standing waves in rotating tires: Experiment and theory, Journal of Sound and Vibration, 227(1999) $1049-1081$

[5] V.H. Saran, K. Ramji, V.K. Goel, Stiffness properties of small-sized pneumatic tyres, Proc. Inst. Mech. Eng., Part D, 216 (2002) 107-114.

[6] J. Padovan, On viscoelasticity and standing waves in tires, Tire Science and Technology, 4(4) (1976) 233-246 
[7] W. Soedel, On the dynamic response of rolling tires according to thin shell approximations, Journal of Sound and Vibration, 41 (1975) 233-246.

[8] W. Kropp, Structure-borne sound on a smooth tire, Applied Acoustics, 26 (1989) 181-192.

[9] R.J. Pinnington, A.R. Briscoe, A wave model for a pneumatic tyre belt, Journal of Sound and Vibration, 253 (2002) 941-959.

[10] J.M. Muggleton, B.R. Mace, M.J. Brennan, Vibrational response prediction of a pneumatic tyre using an orthotropic two-plate wave model, Journal of Sound and Vibration, 264 (2003) 929-950.

[11] J. Perisse, A study of radial vibrations of a rolling tyre for tyre-road noise characterisation, Mechanical Systems and Signal Processing, 16 (2002) 10431058.

[12] L. Fryba, Vibrations of Solids and Structures Under Moving Loads,. $3^{\text {rd }}$ ed., Thomas Telford, London (1999).

[13] D.J. Maglieri, K.J. Plotkin, Sonic boom, In: Aeroacoustics of Flight Vehicles, Ed. H.H. Hubbard, AIP Publishing, V.1 (1995) 519-561. 
[14] V.V. Krylov, A.R. Dawson, M.E. Heelis, A.C. Collop, Rail movement and ground waves caused by high-speed trains approaching track-soil critical velocities, Proc. Inst. Mech. Eng., Part F, 214 (2000) 107-116. 


\section{Figure captions}

Fig. 1. Diagram of an infinite tensioned beam on an elastic foundation: $P$ is the magnitude of the force, $c$ is its velocity, $S$ is the beam tension, $k$ is stiffness of the elastic foundation

Fig. 2. Calculated form of a tyre standing wave generated at super-critical speed condition

Fig. 3. Polar plot illustrating the standing waveform on a tyre circumference

Fig. 4. Calculated forms of a tyre standing wave for different values of vehicle supercritical speed: $352.8 \mathrm{~km} / \mathrm{h}$ (solid line), $432 \mathrm{~km} / \mathrm{h}$ (dashed line), $511.2 \mathrm{~km} / \mathrm{h}$ (dotted line)

Fig. 5. Calculated forms of a tyre standing wave at the vehicle super-critical speed of $352.8 \mathrm{~km} / \mathrm{h}$ for different values of normalised damping: $\beta=0.1$ (solid line), $\beta$ $=0.2$ (dashed line), $\beta=0.3$ (dotted line)

Fig. 6. Critical speed $c_{c r i t}$ as a function of tyre pressure 
Fig. 7. Velocities $c_{c r i t}$ (solid line), $c_{m e m}$ (dashed line) and $c_{c r}$ (dashed line with wider gaps) as functions of tyre pressure

Fig. 8. Critical speed $c_{c r i t}$ as a function of tread thickness

Fig. 9. Velocities $c_{c r i t}$ (solid line), $c_{m e m}$ (dashed line) and $c_{c r}$ (dashed line with wider gaps) as functions of tread thickness 


\section{Table captions}

Table 1. Tyre parameters used for calculations 


\section{Figures}

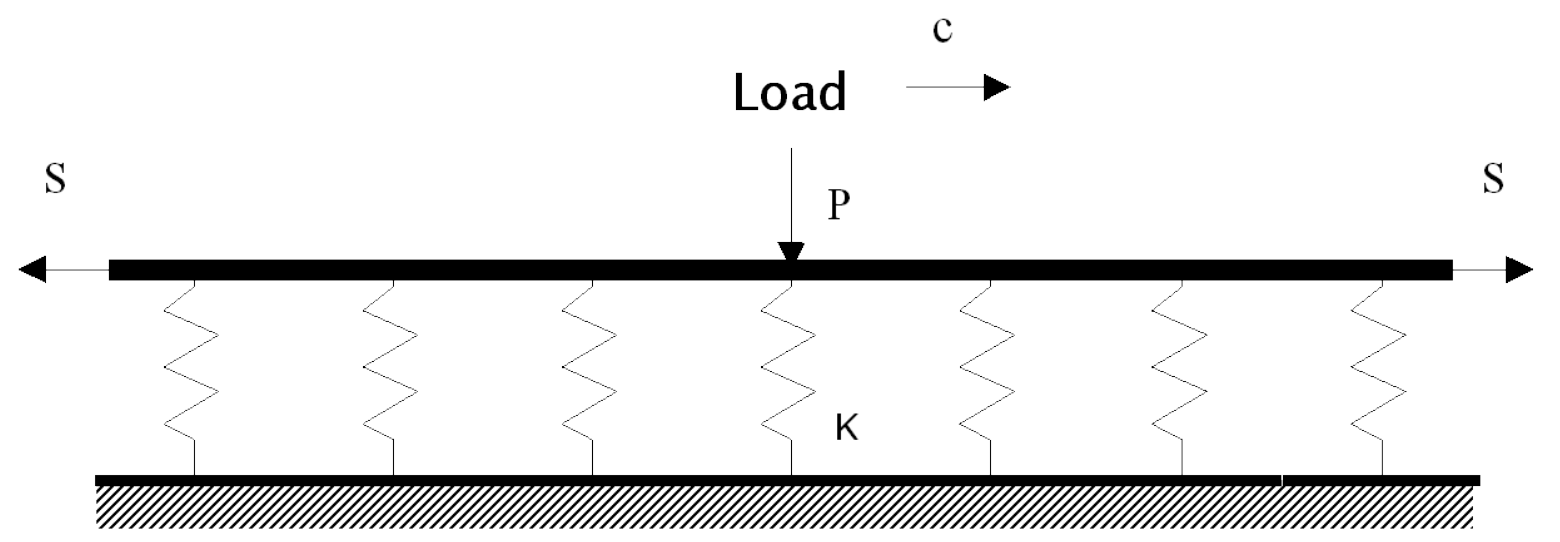

Fig. 1. Diagram of an infinite tensioned beam on an elastic foundation: $P$ is the magnitude of the force, $c$ is its velocity, $S$ is the beam tension, $k$ is stiffness of the elastic foundation 


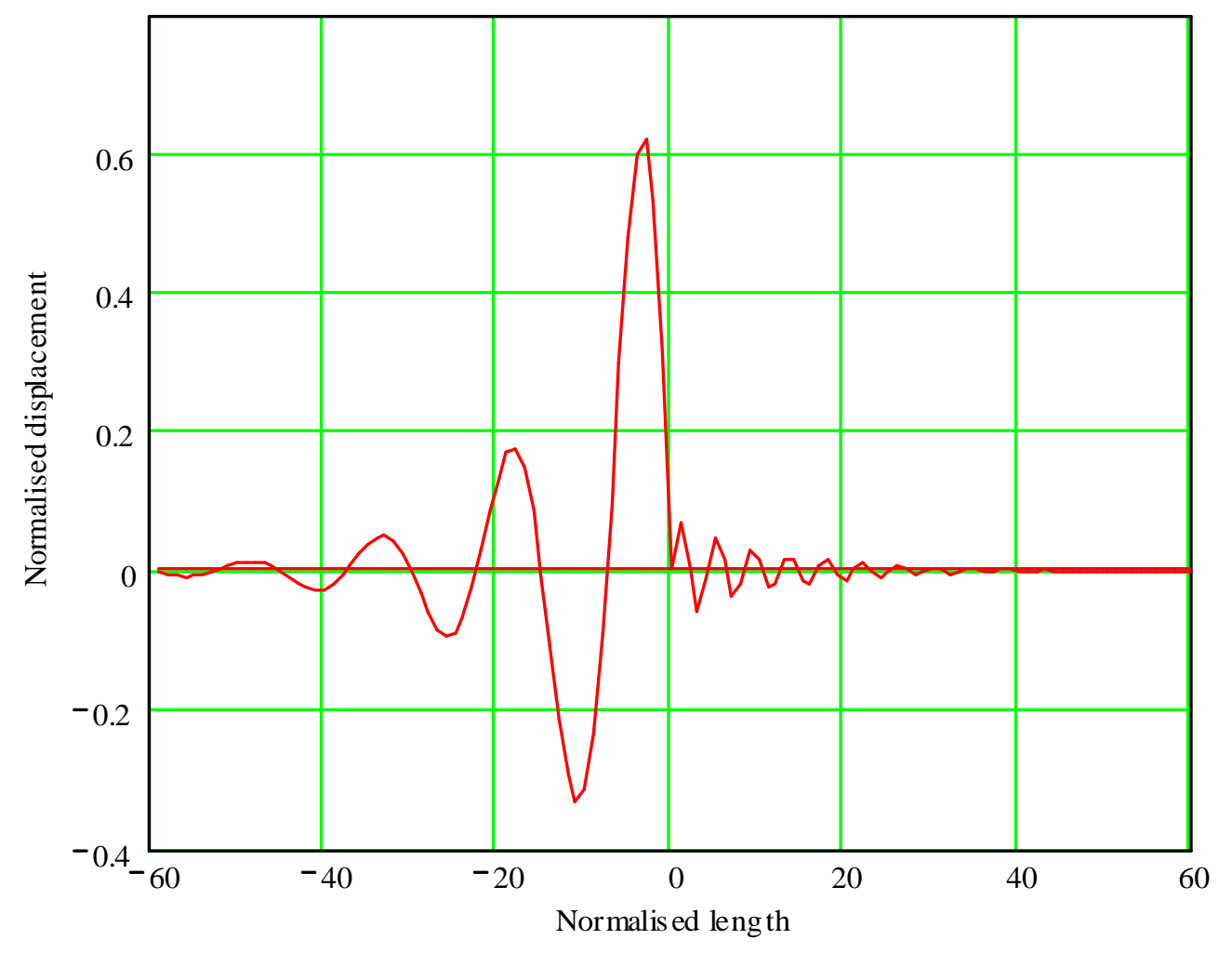

Fig. 2. Calculated form of a tyre standing wave generated at supercritical speed condition 


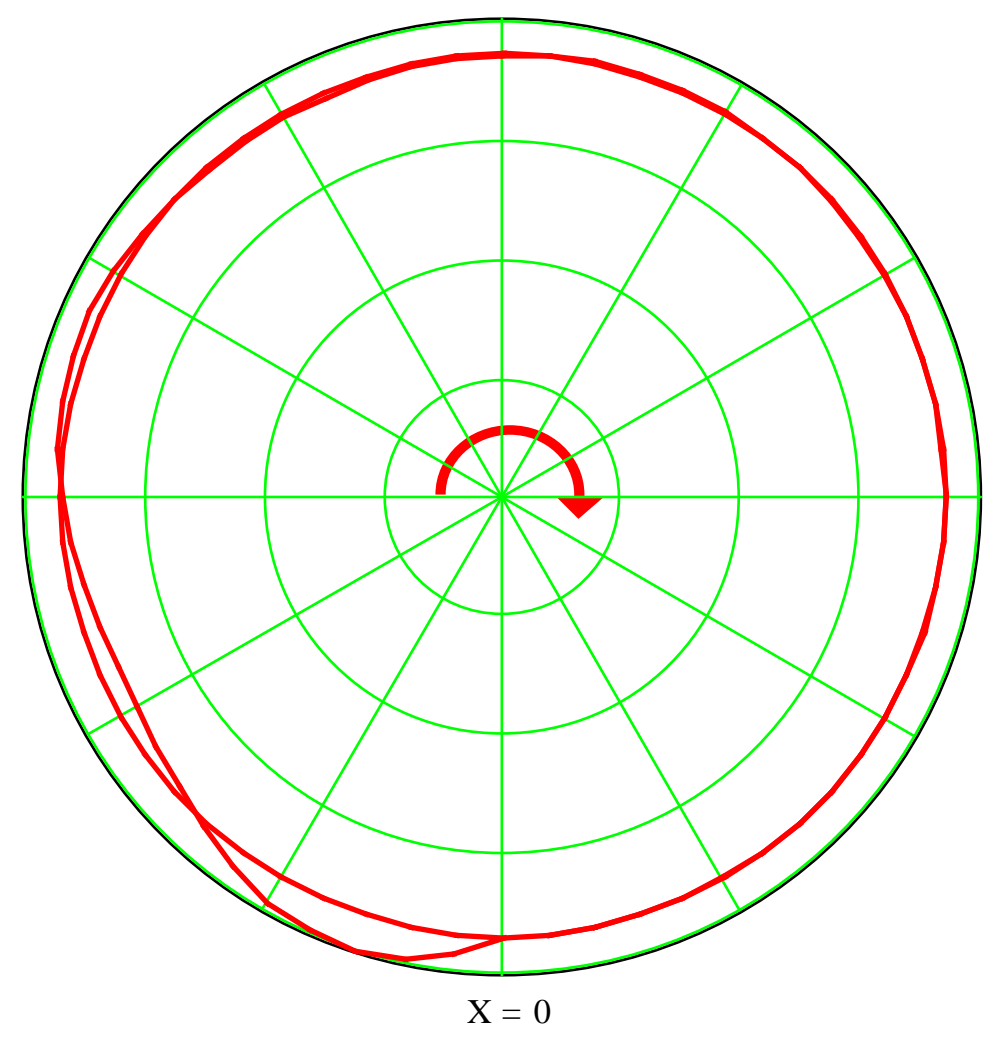

Fig. 3. Polar plot illustrating the standing waveform on a tyre circumference 


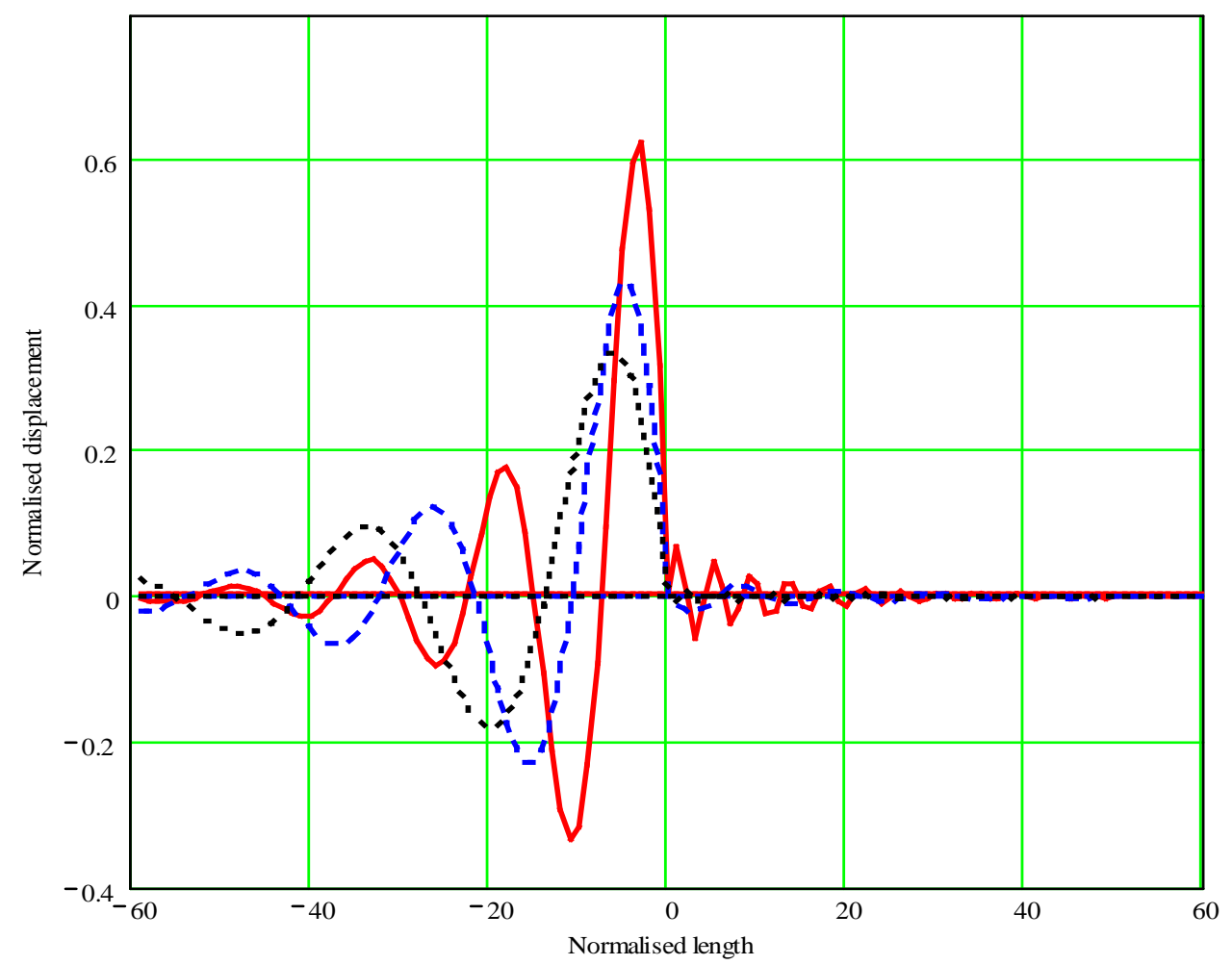

Fig. 4. Calculated forms of a tyre standing wave for different values of vehicle super-critical speed: $352.8 \mathrm{~km} / \mathrm{h}$ (solid line), $432 \mathrm{~km} / \mathrm{h}$ (dashed line), $511.2 \mathrm{~km} / \mathrm{h}$ (dotted line) 


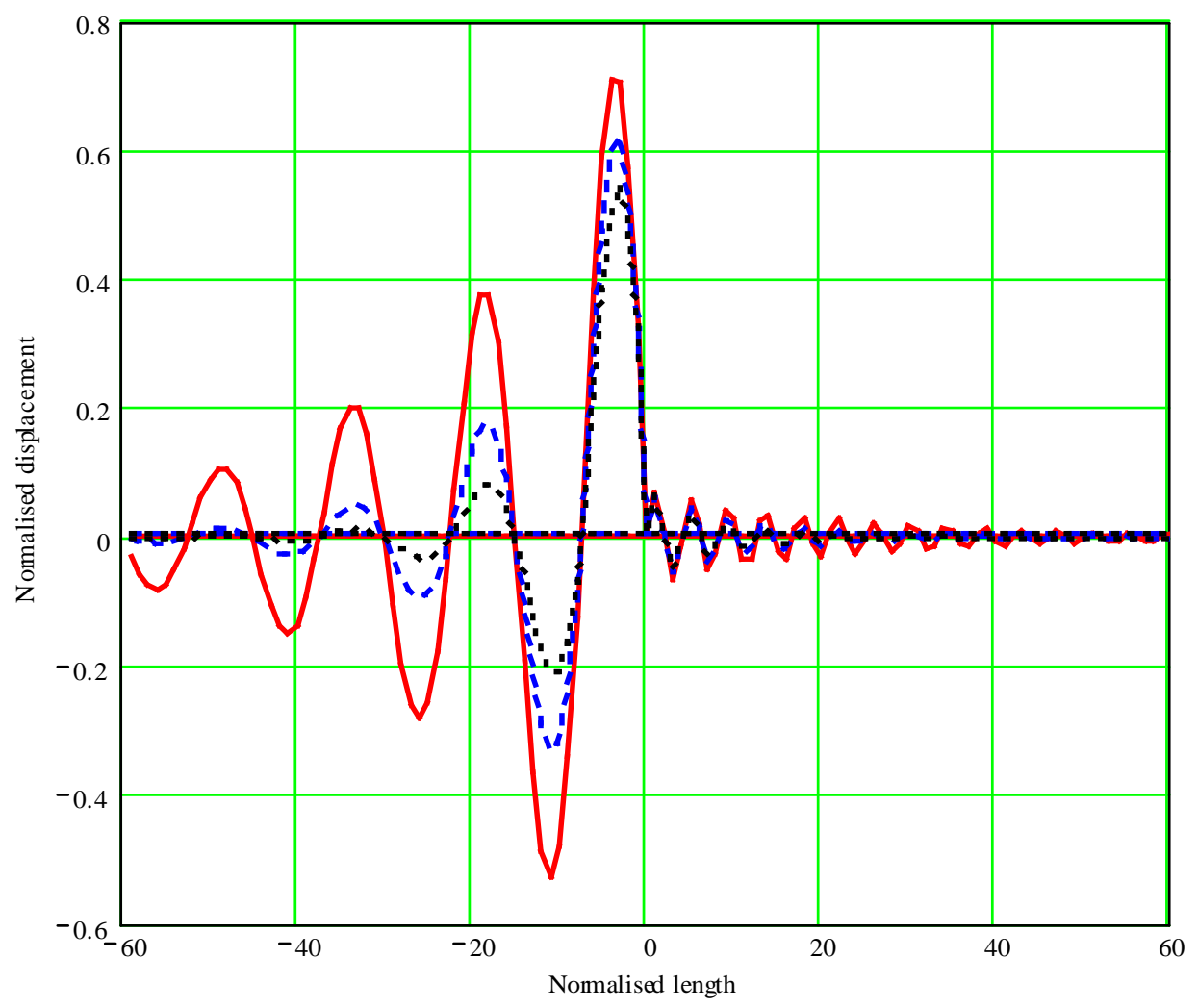

Fig. 5. Calculated forms of a tyre standing wave at the vehicle supercritical speed of $352.8 \mathrm{~km} / \mathrm{h}$ for different values of normalised damping: $\beta=0.1$ (solid line), $\beta=0.2$ (dashed line), $\beta=0.3$ (dotted line) 


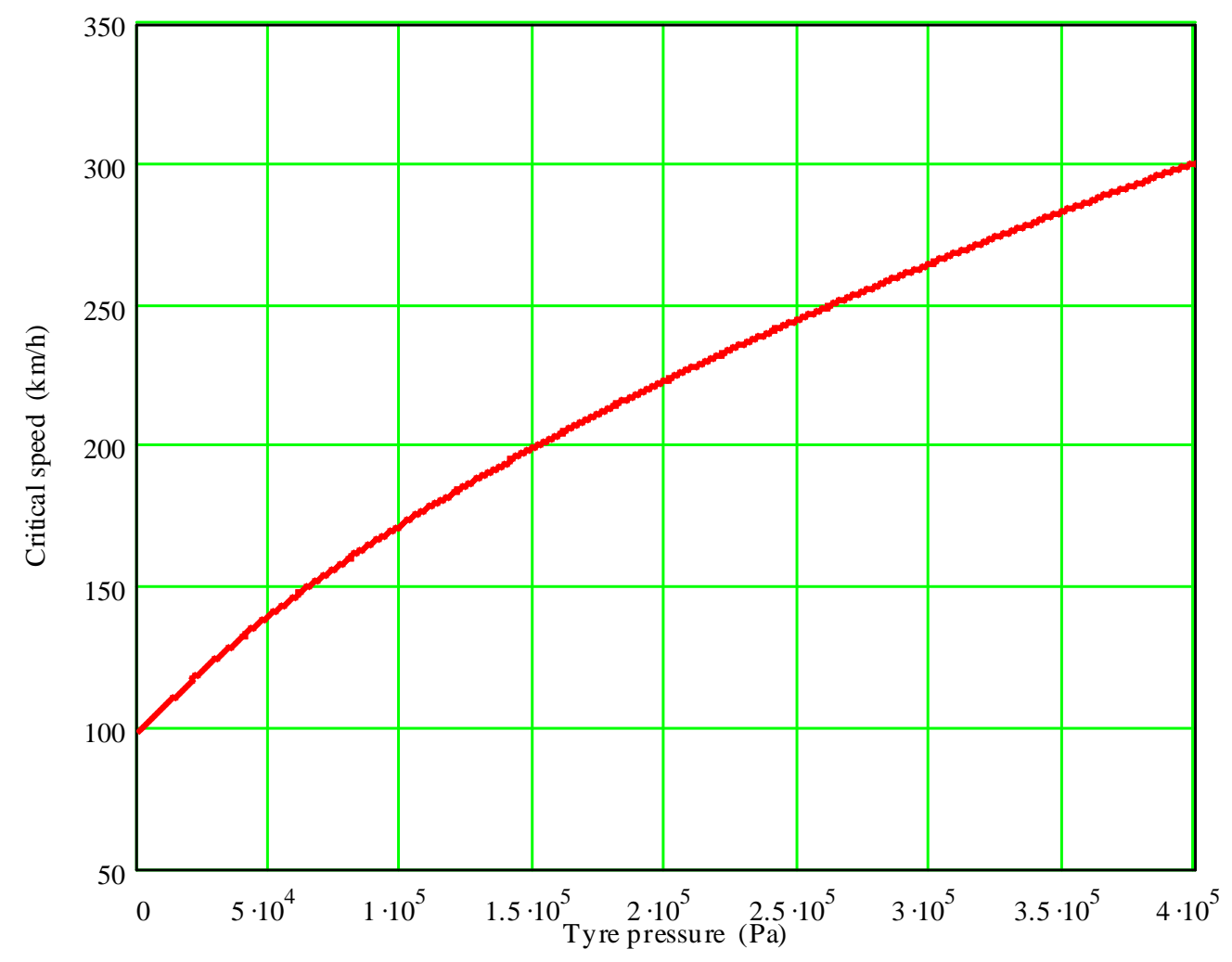

Fig. 6. Critical speed $c_{c r i t}$ as a function of tyre pressure 


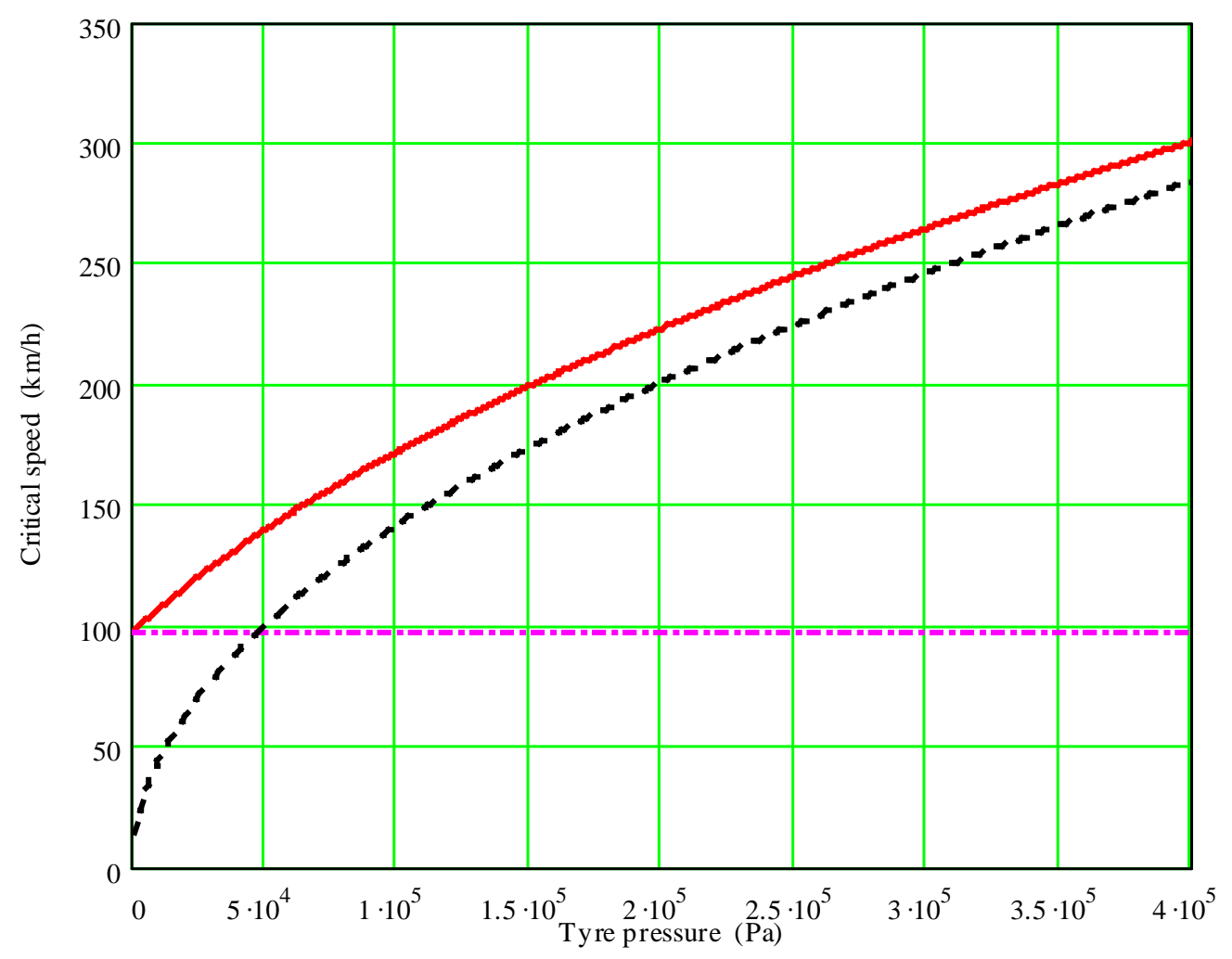

Fig. 7. Velocities $c_{c r i t}$ (solid line), $c_{m e m}$ (dashed line) and $c_{c r}$ (dashed line with wider gaps) as functions of tyre pressure 


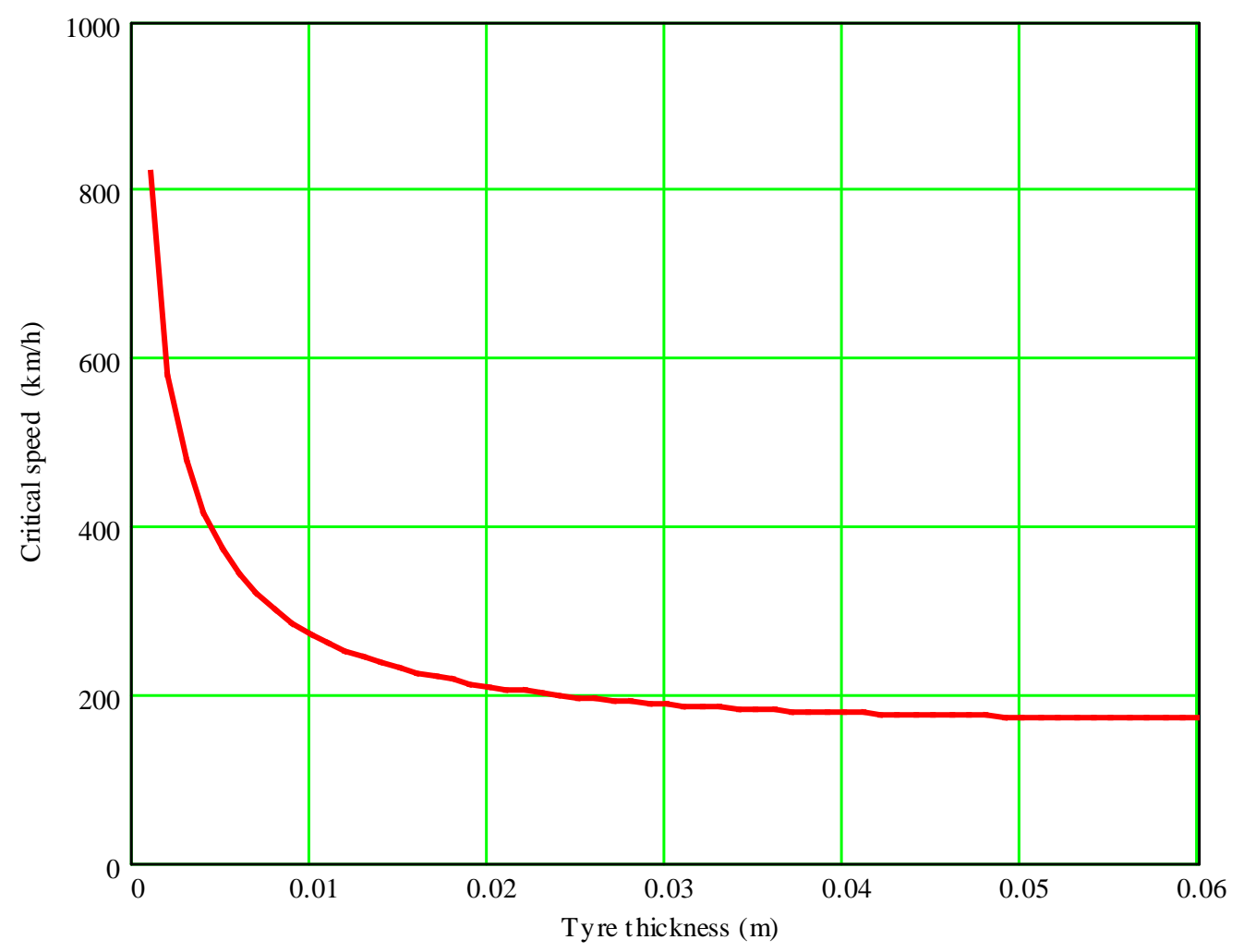

Fig. 8. Critical speed $c_{c r i t}$ as a function of tread thickness 


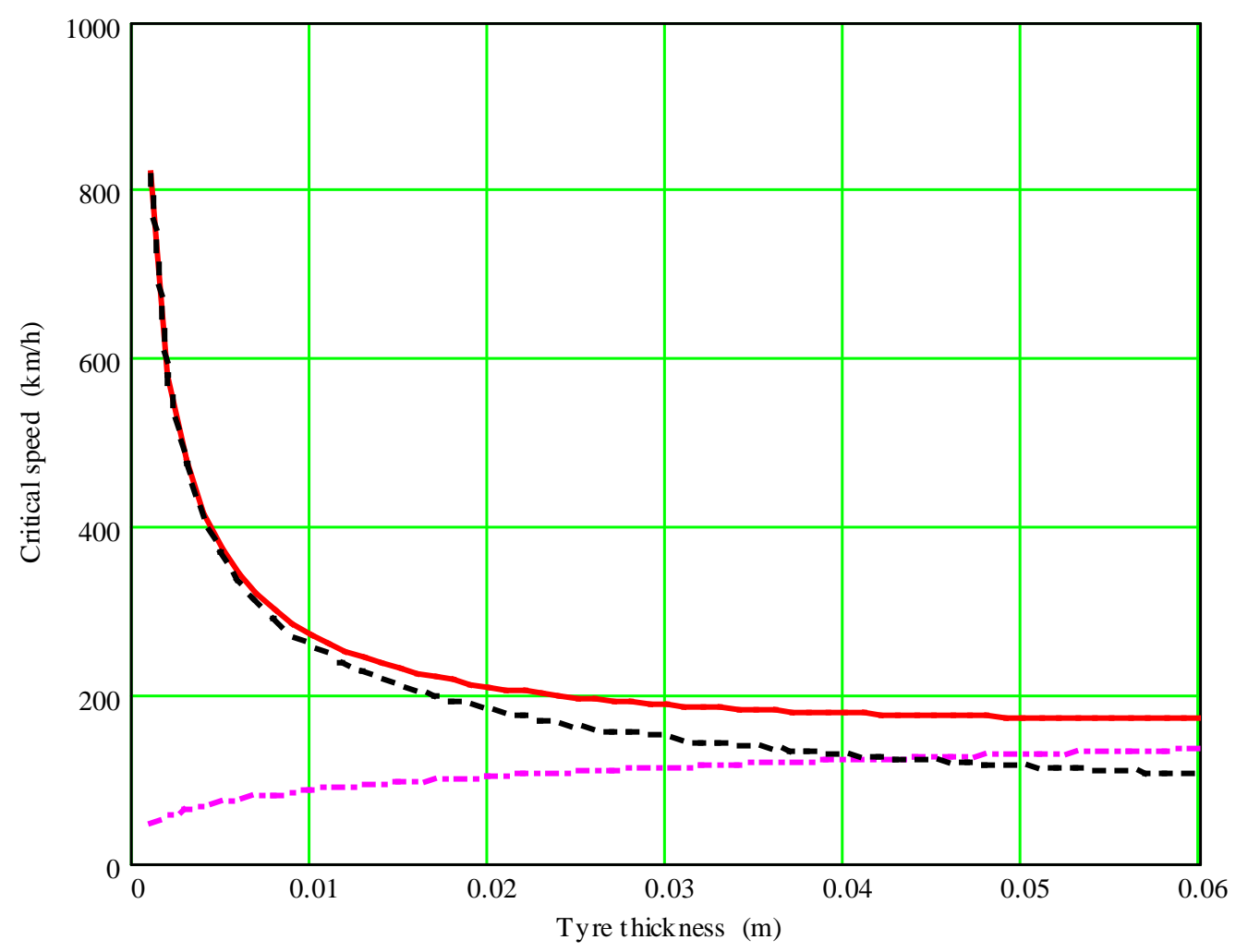

Fig. 9. Velocities $c_{c r i t}$ (solid line), $c_{m e m}$ (dashed line) and $c_{c r}$ (dashed line with wider gaps) as functions of tread thickness 


\section{Tables}

\begin{tabular}{|l|l|l|c|}
\hline Parameter & Notation & Value & Source \\
\hline Tyre radius & $R$ & $0.3 \mathrm{~m}$ & {$[10]$} \\
\hline Belt width & $b$ & $0.2 \mathrm{~m}$ & {$[10]$} \\
\hline Belt thickness & $h$ & $0.016 \mathrm{~m}$ & {$[10]$} \\
\hline Young's modulus & $E$ & $3 \cdot 10^{7} \mathrm{~N} / \mathrm{m}^{2}$ & {$[10]$} \\
\hline Stiffness of foundation & $k$ & $0.97 \cdot 10^{6} \mathrm{~N} / \mathrm{m}^{2}$ & {$[8]$} \\
\hline Mass density & $\rho$ & $1.2 \cdot 10^{3} \mathrm{~kg} / \mathrm{m}^{3}$ & {$[10]$} \\
\hline Beam damping factor & $\beta$ & 0.2 & \\
\hline
\end{tabular}

Table 1. Tyre parameters used for calculations 